\title{
Nacimiento y ocaso de la Federación de Centro América: entre la realidad y el deseo
}

\author{
Guillermo VÁzQuez Vicente \\ Universidad Rey Juan Carlos \\ guillermo.vazquez@urjc.es
}

Recepción: 1 de junio de 2010 / Revisión: 10 de marzo de 2011

Aceptación: 18 de marzo de 2011 / Publicación: diciembre de 2011

\section{RESUMEN}

Centroamérica nace como concepto político pleno de significación en noviembre de 1824 bajo el nombre de Federación de Centro América. Así, tras casi tres siglos de modelo colonial conseguía la independencia de la corona española. Sin embargo, ¿fue este proceso similar al protagonizado por sus vecinos latinoamericanos? El objetivo de este trabajo es analizar el contexto previo y posterior a su nacimiento para intentar dar luz acerca de su repentino fracaso como proceso de integración regional.

Palabras clave: Federación de Centro América, modelo colonial, proceso de independencia, Plan de Iguala, Constitución de la República Federal, Centroamérica, siglo XIX.

\section{Born and decline of Federación de Centro América: beetwen reality and desire}

\begin{abstract}
Central America was born as a full political concept on November 1824 by the name of "Federación de Centro América", achieving the independent from the Spanish empire after almost three centuries under colonial rules. However, ¿was this process similar to the one carried out by the rest of Latin American countries? The aim of this paper is to give light about the previous and subsequent context that could favour its sudden failed as a regional project of integration.
\end{abstract}

Keywords: Federación de Centro América, colonial rules, independence process, Plan de Iguala, Constitución de la República Federal, Centroamérica, 19th Century.

SUMARIO: 1. Introducción. 2. De la proclama independentista al Plan de Iguala: ¿emancipación condicionada? 3. La proclama independentista y las elites regionales: ¿estrategia para mantener el poder? 4. El nacimiento de la Federación de Centro América: ¿utopía o realidad? 5. Conclusiones. 6. Referencias bibliográficas.

\section{INTRODUCCIÓN}

La intención de este trabajo es presentar una radiografía general de la situación histórica que acontecía en el istmo centroamericano en el momento inmediatamente 
anterior y posterior a la formación de la Federación de Centro América ${ }^{1}$. Consideramos que este episodio histórico es de notable importancia debido a que será en el mismo cuando se sentarán las bases de la proclama independentista en la región, a la par que se dará inicio al proceso económico y político que llevará finalmente a las antiguas provincias del Reino de Guatemala al contexto económico y social que determinará su caracterización como naciones soberanas.

Así, cuando fue declarada la independencia de las provincias del antiguo Reino, la satisfacción y el fervor independentista se desbordaron como proclama de un futuro alentador y libre de las ataduras impuestas por un imperio español ya vetusto y en claro declive. Este sentir libertario se unió a una nueva identidad criolla que se desmarcaba del elitista nacionalismo imperial, y creaba una nueva estructura social especialmente gestada por guatemaltecos, salvadoreños y hondureños ${ }^{2}$. No es de extrañar que fueran las firmas de los mandatarios de estos tres países las que aparecieron en el Acta de Independencia suscrita el 15 de septiembre de 1821. Ahora, debido a las limitaciones ocasionadas por las deficientes comunicaciones que presentaba la región en esta primera mitad del siglo XIX, los pueblos nicaragüense y costarricense no tuvieron noticias de este alentador suceso hasta semanas después, y no se suscribieron a la misma hasta ya entrado el mes siguiente ${ }^{3}$. Finalmente este episodio conduciría a la conformación de la Asamblea Constituyente de noviembre de 1824, en la cual se promulgaría la Constitución Federal que serviría como base legal para la creación de la Federación de Centro América.

Pero, ¿cuáles fueron las razones principales que llevaron a las provincias centroamericanas a buscar con tanto ímpetu su proclamación como provincias independientes? ¿Realmente este alzamiento supuso la satisfacción de los intereses de todos los grupos sociales que se daban en ese momento en la región? ¿Fue la Federación de Centro América una solución a los problemas de la región, o bien un instrumento institucional más que posibilitaba el mantenimiento en el poder de las élites dominantes?. Para concluir, ¿hubiese sido posible convertir a la Federación en una realidad?. Estas y otras cuestiones que se irán planteando a lo largo del texto serán las que nos servirán como eje central para la comprensión de este interesante episodio de la historia centroamericana, y las que igualmente nos ayudarán a corroborar la hipótesis central de la que partimos: no existía en esos momentos una correspondencia real

${ }^{1} \mathrm{El}$ autor desea agradecer a la profesora de la Universidad Rey Juan Carlos, Marta Santos, las muchas y valiosas sugerencias realizadas a una versión preliminar de este trabajo. No obstante, cualquier error es responsabilidad exclusiva del autor.

2 Meléndez, 1970; Martínez, 1979; Marroquín, 1964; Dalton, 1984; Pinto, 1986; Hall y Pérez, 2003; Pérez, 2000; Solórzano, 1993; Facio, 1965; y fundamentalmente: DemyK, 1995; Collado, et al., 1986; Anna, 2003; Martinez, s. f.; Soto-Quirós, 2006.

${ }^{3}$ Esta fue la razón por la que hasta el 29 de octubre no tuviese lugar el alzamiento militar en San José ni se diese por concluida la firma de dicha Acta (CAÑAS-DinARTE, 2002, p. 6). Llama la atención que MARure (1960, pp. 73 y ss.) no atribuya la diferencia de fechas a esta cuestión, deduciéndose de su lectura que los intendentes de estas provincias prefirieron esperar y mantenerse neutrales hasta que las convulsiones ocasionadas por la firma de la independencia se calmasen, y los sucesos señalasen el rumbo a seguir. FACIO (1965, pp. 73 y ss.) es también de esta opinión pero dando notable importancia a lo planteado por CAÑAs-DinarTe, 2002. 
entre las condiciones materiales y el ideal plasmado en la Constitución Federal. En consecuencia, ésta será la razón principal que imposibilitó el éxito de la Federación como herramienta de integración del istmo. Pero antes de entrar directamente en esta cuestión, volvamos al contexto histórico previo a la independencia para analizar las razones que pudieron llevar a las provincias del Reino de Guatemala a la independencia definitiva.

\section{DE LA PROCLAMA INDEPENDENTISTA AL PLAN DE IGUALA: ¿EMANCIPACIÓN CONDICIONADA?}

Comencemos nuestro análisis tratando de dar luz acerca de la primera de las cuestiones planteadas: las razones que llevaron a las provincias centroamericanas a buscar su independencia. Las posibles respuestas son múltiples, empero podrían ser sintetizadas en el conjunto que describimos a continuación.

En primer lugar, la debilidad latente del imperio español, que vio perdido su poder naval en el Atlántico a manos británicas ${ }^{4}$, permitió el ingreso regular de productos de los asentamientos británicos en los circuitos comerciales centroamericanos, y ocasionó que a mediados del S. XVIII el comercio de este país controlase en gran medida el comercio exterior de las actuales Honduras, Nicaragua y Costa Rica. Esta cuestión implicó que a finales del período colonial los comerciantes de Belice -ya como principal puerto comercial caribeño- tuviesen bajo su dominio la práctica totalidad del comercio de la región ${ }^{5}$. Con todo, el monopolio inglés en el istmo se tenía que consolidar, y la imposición de un férreo bloqueo comercial a todo el continente europeo ayudó al colapso del comercio entre España y sus colonias, ocasionando que el continente americano se viese inundado de productos de origen británico y norteamericano.

Por otro lado, la situación económica de la región en ese momento era de fuerte crisis al no existir productos exportables rentables ni propuestas de desarrollo comercial ${ }^{6}$. Dicho contexto empeoró en el momento en que el coste de la guerra con Inglaterra comenzó a superar de manera alarmante los escasos ingresos fiscales del Reino de Guatemala. De igual modo, desde mediados del siglo XVII el añil, solo o junto a la grana o cochinilla, había desplazado al maíz, al trigo y fundamentalmente al cacao como producto de exportación principal regional, sometiendo a la minería hondureña a un segundo lugar en los intereses económicos del istmo a causa de los elevados y poco productivos costes de su extracción ${ }^{7}$. Este colorante, conocido también como índigo o jiquilite, era cultivado en las costas del Pacífico, pero tenía su

\footnotetext{
${ }^{4}$ Desastre de la Armada Invencible y batalla de Trafalgar.

${ }^{5}$ Solórzano, 1993, pp. 41 y ss.

${ }^{6}$ Pérez, 2000; Lindo-Fuentes, 1993a y 1993b.

${ }^{7}$ Por su parte, la producción textil guatemalteca fue presa del libre mercado para beneficio de las importaciones de origen británico que, desde el actual Belice, la costa Mosquitia y Jamaica, inundaban en ese momento Centroamérica. De hecho, en 1795 se calculaba que había cerca de mil telares en la ciudad de Antigua Guatemala, mientras que en 1820, cuando ya la producción había mermado, funcionaban 637 telares en la nueva Ciudad de Guatemala, Solórzano, 1993, p. 37.
} 
centro neurálgico en El Salvador. Ahora, a pesar de que la producción estaba dominada en una tercera parte por grandes productores salvadoreños, éstos debían ceder ante el monopolio cerrado de los mercaderes guatemaltecos, que se encargaban de su comercialización, así como de todas las importaciones provenientes de Cádiz, México, Filipinas y Perú ${ }^{8}$. No obstante, debido a que los precios ofertados por Guatemala generalmente no compensaban su cultivo, los grandes productores comenzaron a pervertir la mezcla con determinadas sustancias para así incrementar el volumen de mercancía ofertada -mermando de modo considerable la calidad del productoEste hecho, junto con las numerosas plagas de langosta que devoraban el arbusto y una elevada expansión de la demanda que provocó que dicho producto se cultivase de manera más competitiva en otras regiones ${ }^{9}$, ocasionó que este resurgir comercial tuviese su auge solamente entre 1760 y 1790 , aproximadamente ${ }^{10}$. Habría así que esperar hasta mediados del siguiente siglo para que en el istmo se comenzase a cultivar el que sería su principal producto de exportación hasta la actualidad, es decir, el café.

El gobierno español estableció en 1803 la denominada consolidación de los vales reales como medida para hacer frente a la urgente necesidad de recursos económicos. Al amparo de esta disposición se confiscaron los bienes de la Iglesia de todas las colonias del continente, exigiendo a su vez que aquellos que tuviesen sus propiedades gravadas con capellanías o cofradías pagasen la totalidad de la deuda. Está medida tan impopular propició que los comerciantes provincianos diesen la espalda a la corona y al comercio con el imperio, y se centrasen en los intercambios con los comerciantes británicos ${ }^{11}$.

Para empeorar aun más la situación, en 1808 se produjo la invasión francesa del territorio español y el apresamiento y exilio a suelo galo del monarca Fernando VII. Este acontecimiento puso en entredicho la independencia española, pues sin flota naval y con el bloqueo británico, se encontraba aislada y en pleno conflicto bélico frente a la invasión francesa. Sin embargo el asunto no quedó aquí, ya que con el vacío de poder propiciado por la no aceptación de José Bonaparte como monarca español, Centroamérica vivió el inicio de su particular transición política de la mano de la élite guatemalteca que, tras las noticias de la guerra de España, tuvo que mantener el poder en todas las provincias del istmo ${ }^{12}$. Para este cometido fueron organizadas milicias, que bajo su control sofocaron los primeros conatos de rebelión que pretendían la

${ }^{8}$ Floyd, 1989; Marroquín, 1964; Dalton, 1984; Woodward, 1989; Vives, 1987; Meléndez, 1970; Fernández, 2003; Palma, 1986; Taracena, 2002.

${ }^{9}$ Venezuela, la India y las Antillas Holandesas fundamentalmente.

${ }^{10}$ A lo anterior hay que añadir la aparición de añilinas o colorantes sintéticos descubiertos por la industria química alemana a mediados del siglo XIX. Torres-Rivas, 1989, pp. 24 y ss.

${ }^{11}$ SolÓRZAno, 1993, p. 64. Este hecho supuso un verdadero desastre económico a nivel regional, pues en plena crisis de exportación del añil más de un millón de pesos fueron enviados hacia España en concepto de pago de vales reales.

${ }^{12}$ No conviene olvidar el monopolio estatal que ostentaba la élite guatemalteca a inicios de este siglo. Por ejemplo, en 1812 se informó de que de los 740 puestos que componían la maquinaria administrativa del Reino de Guatemala, casi 700 se encontraban en poder de empleados de esta región. Informe reservado del presidente de la Audiencia de Guatemala José de Bustamante, 30 de enero de 1821. AGI/AC. Leg. 631. Citado por Pinto, 1993, p. 77. 
emancipación comercial de las provincias con respecto al monopolio guatemalteco ${ }^{13}$. No obstante, las guerras por la independencia que desde aproximadamente 1810 se daban en México y toda Sudamérica encenderían la mecha que concluiría con la independencia definitiva de todas las naciones del continente ${ }^{14}$.

Para concluir, en el mismo plano que las anteriores, es importante dejar constancia acerca de la guerra de independencia norteamericana, concluida en 1776, la revolución francesa de 1789 y la utopía reformista de las Cortes de Cádiz de 1812. Dichos procesos inspiraron el surgimiento de una serie de ideas libertarias y humanistas que sin duda alentaron a los grandes pensadores centroamericanos en su ideal independentista. Concretamente, el fracaso de las Cortes de Cádiz, que sobrevino tras la reinstauración del absolutismo español en 1814, fue un duro golpe para los reformistas centroamericanos que veían en esta utopía del constitucionalismo un triunfo de la España ilustrada que abriría posibilidades para una nueva alianza, bajo principios liberales, entre colonia y metrópoli ${ }^{15}$.

Es decir, a pesar de que acabamos de mostrar sólo un bosquejo de lo que comúnmente se suele argumentar acerca de las posibles causas que iniciaron, potenciaron o agudizaron -según distintas fuentes- el proceso de independencia centroamericano, podemos afirmar que a lo largo de la segunda mitad del siglo XVIII la región fue entrando en un contexto de crisis generalizada que se concretó en un evidente deterioro de sus relaciones con la metrópoli. Persistían igualmente los problemas comerciales y sociales que se agravaron al entrar en decadencia la producción y exportación del añil en el caso de la primera, y al iniciarse en la región los primeros alzamientos populares fruto de las ideas liberales en el caso del segundo.

Sin embargo, y volviendo al 15 septiembre de 1821, es conveniente señalar que a pesar de que el proceso de independencia centroamericano nace con el Acta de Independencia suscrita en esta fecha, ésta no vino acompañada de una proclamación real de sus provincias como naciones independientes, sino que fue consecuencia directa de la invitación que el gobierno mexicano hizo a las autoridades centroamericanas para adherirse al Plan de Iguala, y que se consumo el 5 de enero de 1822 con el Acta de Unión de las Provincias de Centro América al Imperio Mexicano ${ }^{16}$. Ahora bien, ¿qué pudo llevar a estas naciones a aceptar la propuesta de Agustín de Iturbide? Esta cuestión nos lleva directamente al segundo interrogante planteado en este trabajo, y

${ }^{13}$ Siendo las más importantes las iniciadas en El Salvador y Nicaragua en 1811. En Marure (1960), SolóRZANO, (1993) y PINTO (1993) se puede encontrar información detallada de este capítulo histórico.

${ }^{14}$ LynCh, 1973 y 2001; Mitre, 1950; Guerra, 1992.

${ }^{15}$ Pérez, 2000, p. 84. A lo anterior, Pinto (1993, p. 84 y ss.) añade la influencia que en el istmo tuvo el levantamiento antiesclavista de la isla de Santo Domingo, que en el caso de Haití llegó a triunfar y consiguió la abolición de la esclavitud. Para más información acerca de la influencia de las Cortes de Cádiz en el contexto político centroamericano se puede consultar: RodríGuez, 1984; Hall y Pérez, 2003; GARCíA, 1994. En GuARISCO, 2007 se pueden encontrar numerosas referencias bibliográficas al respecto.

${ }^{16}$ Wortman, 1976; Benson - Berry, 1969; Anna, 1991; LujÁn, 1982; Toussaint, et al., 2001. Para información acerca del proceso de independencia mexicano se pueden consultar las obras de FrASQUET, 2007 y Chust - Serrano, 2007, que desde un punto de vista historiográfico analizan esta cuestión dando importantes referencias bibliográficas. 
su respuesta tiene una posible explicación en el juego de poder político que desarrollaba la élite del istmo.

\section{LA PROCLAMA INDEPENDENTISTA Y LAS ELITES REGIONALES: ¿ESTRATEGIA PARA MANTENER EL PODER?}

Desde los levantamientos de 1811 la clase dominante centroamericana se encontraba aterrada con la posibilidad de que en el seno de la región se pudiese dar un alzamiento popular articulado. Así, a pesar de que los grupos independentistas que habían impulsado dichos acontecimientos se encontraban completamente aislados y debilitados a causa del aparato represor del gobierno colonial, desde el comienzo de las guerras de independencia que se daban en todo el continente y la proclamación de las Cortes de Cádiz, en toda la región -incluida Guatemala- dio comienzo un nuevo movimiento republicano que entre 1820 y 1821 buscó la forma de organizarse a nivel nacional ${ }^{17}$. Por consiguiente, desde que comenzó a circular la noticia de la posible anexión al imperio mexicano, está facción inició su propio proceso de independencia mediante el alzamiento militar.

Ahora bien, por inferencia de lo anterior la oligarquía era plenamente consciente de que tras el éxito del Plan de Iguala y de las guerras de independencia que asolaban Sudamérica, el antiguo Reino de Guatemala no podía continuar bajo condiciones político-administrativas coloniales. Estos hechos, junto con el mencionado auge republicano que se hacía sentir ya en toda la región, convenció definitivamente a la aristocracia colonial de la necesidad de proclamar la independencia para así tener la posibilidad de seguir conservando en sus manos el poder político. De este modo, en el Acta de Independencia se recoge que:

Siendo públicos é indudables los deseos de independencia del gob ${ }^{\circ}$ Español q. por escrito y de palabra ha manifestado el pueblo de esta Capital: recibidos por el ultimo Correo diversos oficios de los Ayuntamtos Constitucionales de Ciudad Real, Comitan y Tuxta, en q. comunican haber proclamado y jurado dha. independencia, y excitan á, qe. se haga lo mismo en esta Ciudad: siendo positivo q. han circulado iguales oficios á otros Ayuntamtos. [... De manera] que siendo la independencia del gobierno Español, la voluntad gral. del pueblo de Guat ${ }^{\mathrm{a}}$, y sin perjuicio de lo q. determine sobre ella el Congreso q. debe formarse, el Sor. gefe Político la mande publicar pa. prevenir las consecuencias q. serian temibles en el caso de q. la proclamase de hecho el mismo pueblo ${ }^{18}$.

${ }_{17}$ Pinto, 1993, p. 92 y ss. Además, a mediados de 1820 se dio un levantamiento indígena en Guatemala que a pesar de ser controlado rápidamente, confirmó el descontento de este sector social. Para más información referente a la situación de este colectivo en el momento de la independencia se puede consultar principalmente: Alda, 2000; Falla, 1971; Bertrand, 1987; Pollack, 2008.

${ }_{18}$ Acta de la Independencia. Guatemala, 15 de septiembre de 1821. Herrarte 1956, Doc no 17, pp. 9-12. Documento digital disponible en la página web del SICA: http://www.sica.int/cdoc/publicaciones/ mem_odeca/sg1/union_ca_odeca_1956.pdf 
Como se puede apreciar, las elites prefirieron proclamar la independencia por el temor, fundamentado en los diferentes alzamientos populares, de que finalmente fuese el mismo pueblo el que mediante un alzamiento definitivo tomase las riendas del proceso. Pero para no llevar a engaño al lector, hemos de aclarar que a pesar de este nuevo rumbo político tanto las provincias como las capitales de la región continuaron gobernadas por la misma elite que proclamó la independencia y que previamente ostentaba el poder en el istmo. De hecho, el general Gabino Gaínza quedó al mando del nuevo gobierno, mientras que la única novedad vino de la mano de la formación de una Junta Provincial Consultiva compuesta por las antiguas autoridades y representantes de la aristocracia colonial centroamericana, cuyo único cometido era el asesoramiento del citado general en cuestiones de índole gubernamental ${ }^{19}$. Por lo tanto, a pesar de que este episodio puede ser considerado como un alentador triunfo de la proclama independentista de las masas, desde cierto punto de vista no representó más que el triunfo de los planes políticos de la oligarquía guatemalteca frente a los intereses reales del conjunto de la sociedad centroamericana ${ }^{20}$.

Por su parte, las oligarquías locales vieron en la proclama independentista y en la propuesta de Iturbide la doble posibilidad de liberarse del tutelaje guatemalteco $\mathrm{y}$, a su vez, de luchar contra el elemento republicano insurgente que amenazaba con imponer sus intereses en el nuevo rumbo de la región. Por este motivo, la ciudad de Comayagua, antigua capital colonial de Honduras, la Diputación Provincial de León en Nicaragua y Cartago en Costa Rica fueron las primeras ciudades que proclamaron su anexión al potente virreinato mexicano, con el objetivo de buscar una solución contundente a dichos problemas.

Ahora, el contenido conservador del Plan de Iguala también reflejaba los intereses de una oligarquía guatemalteca que veía peligrar su poder en el istmo a causa del contexto social de inminente alzamiento, tanto en lo concerniente a la masa social como en lo relativo a las élites locales. Por este motivo percibió esta propuesta como un instrumento para mantener la estabilidad, ya que no podemos dejar de lado el potencial disuasorio que el ejército mexicano representaba para la sociedad centroamericana ${ }^{21}$.

19 “Que el Sr. Gefe Politico Brigadier Dn. Gavino Gainza, continúe con el Gobierno Supor. Político y Militar, y $\mathrm{p}^{\mathrm{a}} \mathrm{q}$. este tenga el caracter q. parece propio de las circunstancias, se forme una junta provisional consultiva, compuesta de los Sres. individuos actuales de esta Diputacion Provincial, y de los Sres. Dn. Miguel de Larreynaga Ministro de esta Audiencia, Dn. José del Valle Auditor de Guerra, Marqués de Aycinena, Doctor Dn. José Valdez, Tesorero de esta Sta. Yglesia, Dr. Dn. Angel M 5 Candina, y Lincend ${ }^{\circ}$ don Antonio Robles, Alcl $3^{\circ}$ constitucional: el prim ${ }^{\circ}$ pr. la Prov ${ }^{\mathrm{a}}$ de Leon, el $2^{\circ}$ pr. la de Comayagua, $3^{\circ}$ pr. Quesaltenango, $4^{\circ}$ pr. Sololá y Chimaltenango, $5^{\circ}$, pr. Sonsonate, y el $6^{\circ} \mathrm{pr}$. Ciudad RI. de Chiapa. [...] Que esta Junta provisional consulte al Sr. Gefe politico en todos los asuntos economicos y gubernativos, dignos de su atención" Ibídem, Numerales 8 y 10, p. 10.

${ }^{20}$ Lynch, 1973; TARACENA, 1995; Torres-Rivas, 1989; Pinto, 1993 (consultar también bibliografía relacionada en la nota 1). En relación con lo anterior, PINTO, 1993, p. 102, se atreve a definirlo como una comedia en dos actos, donde el primero fue la proclamación de la independencia de septiembre de 1821 por parte de estos intereses elitistas y el segundo la posterior anexión a México. Por otro lado, Pollack, 2009, considera que más que una demostración del poder de la oligarquía, la proclamación de la independencia en Guatemala puede ser considerada como una debilidad.

${ }^{21}$ PINTO, 1993, p. 95 y ss. Además, el clero jugó un papel fundamental en este proceso al transformar la anexión en un problema religioso y calificar de herejes a las facciones contrarias. Así mismo, para 
En consecuencia, en el Acta de Unión de las Provincias de Centro América al Imperio Mexicano se recogía que:

Entre las varias concideraciones que ha hecho la Junta, en esta importante y grave materia, en que los pueblos se hayan amenazados en su reposo, y especialmente en la unión con sus hermanos de las otras provincias con quienes ha vivido siempre ligados por la vecindad, comercio y otros vínculos estrechos, fue una de las primeras, que por medio de la unión a Mexico querían salvar la integridad de lo que antes se ha llamado Reino de Guatemala, y restablecer entre si la unión que ha reinado por lo pasado; no apareciendo otro para remediar la divición que se experimenta. [...Y] como algunos pueblos han fijado al juicio de la Junta lo que más les convenga resolver en la presente materia y circunstancias, por no tenerlas todas a la vista; la Junta juzga, que manifestada, como está de un modo tan claro, la voluntad de la universalidad, es necesario que los dichos pueblos se adhieran a ella para salvar su integridad y reposo ${ }^{22}$.

Expresado de otro modo, se determinaba que el alegato unionista era la única vía para solucionar los problemas segregacionistas del istmo, planteando a su vez la necesidad de que aquellos pueblos que hubieran expresado algún tipo de duda acerca de esta cuestión, se adhiriesen a la invitación de Agustín de Iturbide para salvaguardar plenamente el concepto geopolítico que representaba el Reino de Guatemala.

Pero este capítulo de adhesión estaba llamado a ser corto, y así la diferencia de intereses dentro de las propias élites regionales, la lejanía del centro de poder y la persistente semilla independentista en forma de conflicto bélico, continuaban espoleando a cualquier nación que pretendiese adherir estas provincias a su reino ${ }^{23}$. En consecuencia, el envío de contingentes militares a la capital de Guatemala para intentar controlar a los insurgentes -en su mayoría guatemaltecos y salvadoreños- no hizo más que intensificar este afán libertario, y después de que una rebelión de generales mexicanos destituyese del cargo a Iturbide, la guerra civil se extendió definitivamente por toda la región. A partir de este momento, Vicente Filísola, el oficial a cargo del destacamento en Guatemala, no pudo más que convocar a un Congreso para discutir el tema de la anexión centroamericana a México.

Este último se concretó el 1 de julio de 1823 con el objetivo de proclamar la independencia absoluta de Centroamérica, y con la intención de declarar a ese Congreso como

Iturbide, muy consciente de la precaria situación interna de Centroamérica, este capítulo significaba la oportunidad de ensanchar las fronteras de su imperio.

${ }_{22}$ Acta de la Unión de las Provincias de Centro América al Imperio Mexicano. Guatemala, 5 de enero de 1822. Extraído de: Herrarte, 1956, pp. 13-16. Documento digital disponible en la página web del SICA: http://www.sica.int/cdoc/publicaciones/mem_odeca/sg1/union_ca_odeca_1956.pdf

${ }^{23}$ De hecho, este afán de independencia había incluso llevado a El Salvädor --que nunca ocultó su republicanismo y rechazo a la anexión a México-- a pedir la adhesión a los Estados Unidos en septiembre de 1822 (PÉrez, 2000, p. 88). Con el propósito de someter a las ciudades rebeldes, y especialmente a la provincia de San Salvador, Iturbide envió a la región un fuerte destacamento. Pero la tradición anticolonial y la derrota del ejército guatemalteco-mexicano de la mano del general Arce, lograron que a lo largo de todo el período de anexión esta provincia permaneciese independiente del monopolio Guatemala-México. 
Asamblea General Constituyente ${ }^{24}$. Comenzaba así el verdadero proceso de emancipación regional, que a través del Decreto de Independencia Absoluta de las Provincias del Centro de América de 19 de julio de 1823, no dejaba lugar a dudas al proclamar:

Que la Independencia del Gobierno español ha sido y es necesaria en las circunstancias de aquella nación y las de toda la América: que era y es justa en sí misma y esencialmente conforme a los derechos sagrados de la naturaleza: que la demandaban imperiosamente las luces del siglo, las necesidades del Nuevo Mundo y todos los más caros intereses de los pueblos que lo habitan. Que la naturaleza misma resiste la dependencia de esta parte del globo separada por un Océano inmenso de la que fue su metrópoli, y con la cual le es imposible mantener la inmediata y frecuente comunicación, indispensable entre los pueblos que forman un solo Estado. Que la experiencia de más de trescientos años manifestó a la América que su felicidad era del todo incompatible con la nulidad a que la reducía la triste condición de colonia de una pequeña parte de la Europa. Que la arbitrariedad con que fue gobernada por la nación española y la conducta que ésta observó constantemente, desde la conquista, excitaron a los pueblos al más ardiente deseo de recobrar sus derechos usurpados. Que a impulsos de tan justos sentimientos, todas las provincias de América sacudieron el yugo que las oprimió por espacio de tres siglos: que las que pueblan el antiguo reino de Guatemala proclamaron gloriosamente su independencia en los últimos meses del año de 1821; y que la resolución de conservarla y sostenerla es el voto general $\mathrm{y}$ uniforme de todos sus habitantes ${ }^{25}$.

Pero además, en claro conflicto con los intereses primeros que marcaron la aceptación por parte de la élite centroamericana de la propuesta de adhesión al Plan de Iguala, en el Decreto se dejaba constancia de que:

[...] La incorporación de estas provincias al extinguido imperio mexicano, verificada sólo de hecho en fines de 821 (sic.) y principios de 822 (sic.), fue una expresión violenta arrancada por medios viciosos e ilegales. Que no fue acordada ni pronunciada por órgano ni por medios legítimos: que por estos principios la representación nacional del estado mexicano, jamás la aceptó expresamente, ni pudo con derecho aceptarla; y que las providencias que acerca de esta unión dictó y expidió D. Agustín de Iturbide, fueron nulas. Que la expresada agregación ha sido y es contraria a los intereses y a los derechos sagrados de los pueblos nuestros comitentes: que es opuesta a su voluntad y que en concurso de circunstancias tan poderosas e irresistibles exigen que las provincias del antiguo reino de Guatemala se constituyan per si mismas y con separación del Estado Mexicano ${ }^{26}$.

${ }^{24}$ Empero, como muestra de la supremacía mexicana y de la debilidad de la oligarquía dominante en el istmo, Filísola retiró sus tropas un mes después de esta fecha llevándose bajo el brazo la adhesión de Chiapas a México. Para más información acerca de este episodio histórico se puede consultar: Belson - Berry, 1989; Avendaño, 2008; Acuña, 2005. Para profundizar en la problemática chiapaneca: CARvalho, 1994; Gutiérrez, 2005.

${ }^{25}$ Decreto de Independencia Absoluta de las Provincias del Centro de América. Guatemala, 11 de julio de 1823. Extraído de: Herrarte, A. 1956, pp. 17-20. Documento digital disponible en la página web del SICA: http://www.sica.int/cdoc/publicaciones/mem_odeca/sg1/union_ca_odeca_1956.pdf

${ }^{26}$ Ibidem, Numeral 2º, pp. 18-19. 
Y por lo tanto, los representantes de las provincias signatarias declaraban:

$1^{\circ}$ - Que las expresadas provincias, representadas en esta Asamblea, son libres e independientes de la antigua España, de México y de cualquiera otra potencia, así del antiguo como del nuevo mundo; y que no son ni deben ser el patrimonio de persona ni familia alguna. $2^{\circ}$ - En consecuencia, son y forman nación SOBERANA, con derechos y en aptitud de ejercer y celebrar cuantos actos, contratos y funciones ejercen y celebran los otros pueblos libres de la tierra. $3^{\circ}$ - Que las provincias sobre dichas, representadas en esta Asamblea (y demás que espontáneamente se agreguen de los que componían el antiguo reino de Guatemala) se Ilamaran, por ahora sin perjuicio de lo que resuelva en la Constitución que ha de firmarse: «PROVINCIAS UNIDAS DEL CENTRO DE AMERICA $\aleph^{27}$.

Es decir, de nuevo la oligarquía tomaba las riendas de la evolución política del istmo y se decantaba por la estrategia que más convenía a sus intereses regionales. Si en un primer momento la pretensión se concretó en una alianza con un imperio cuya hegemonía militar ayudaba a mantener el orden social y las pretensiones unionistas, en este caso el entorno de marcado conflicto bélico dejaba como única vía de unificación regional la independencia plena de Centroamérica de cualquier imperio. Pero en un contexto de guerra civil, ¿de qué manera pretendía la oligarquía mantener sus privilegios? Es hora de entrar a analizar las consecuencias de esta decisión.

\section{EL NACIMIENTO DE LA FEDERACIÓN DE CENTRO AMÉRICA: ¿UTOPÍA O REALIDAD?}

Si bien con la instauración de este primer Congreso Constituyente se proclamaron las Provincias Unidas del Centro de América, toda la labor de la Asamblea concluyó el 22 de noviembre de 1824 con la promulgación de la Constitución de la República Federal de Centro-América y la denominación de la República como Federación de Centro América ${ }^{28}$. En lo relativo a la organización del poder se determinaba que el

\footnotetext{
${ }^{27}$ Ibídem, p. 19.

${ }^{28}$ Constitución de la Republica Federal de Centro-América, dada por la Asamblea Nacional Constituyente en 22 De Noviembre de 1824. Guatemala, 22 de noviembre de 1824. Extraído de: Herrarte, 1956, pp. 29-58. Documento digital disponible en la página web del SICA: http://www.sica. int/cdoc/publicaciones/mem_odeca/sg1/union_ca_odeca_1956.pdf. En ésta, claramente influenciada por la constitución estadounidense y por las Cortes de Cádiz, se establecía que "el pueblo de la República federal de Centro América es soberano é independiente" (Art.1), siendo su "primer objeto la conservación de la libertad, igualdad, seguridad y propiedad" (Art.2). De igual modo se remarcaba que "el gobierno de la República [sería] popular, representativo [y] federal" (Art.8), y que dicha República pasaba a denominarse Federación de Centro América (Art.9). Finalmente se declaraba que "la federación [quedaría compuesta] de cinco estados que son: Costarrica, Nicaragua, Honduras, el Salvador y Guatemala", mientas que "la provincia de Chiapas se tendrá por estado de la federación cuando libremente se una" (Art.6). A este respecto, en virtud de los Artículos 196, 197 y 198, el 2 de febrero de 1838 la provincia de Los Altos pasaría a ser el sexto Estado de la Federación. Sobre la provincia de Los Altos se puede consultar TARACENA, 1997.
} 
poder legislativo residiría en el Congreso -compuesto por representantes popularmente elegidos en razón de uno por cada 30.000 habitantes-, siendo sus atribuciones la de hacer las leyes y la de autorizar al poder ejecutivo para emplear la milicia de los estados cuando lo exija la ejecución de la ley, o sea necesario contener insurrecciones o repeler invasiones. Lo que nos lleva directamente al papel del poder ejecutivo, el cual sería ejercido por un Presidente nombrado por el pueblo por un período de cuatro años, pudiendo ser reelegido una vez sin intervalo alguno. Pero el matiz a destacar era que el Presidente tendría a su mando toda la fuerza armada de la Federación, y que además podría usarla para repeler invasiones o contener insurrecciones, dando siempre cuenta al Congreso, o en su receso al senado.

Es decir, la decisión final de actuación de las fuerzas armadas recaía en el Congreso. Pero, ¿qué composición presentaba en ese momento esta institución? En función de los habitantes de cada provincia, el Primer Congreso Federal -abril de 1825quedó conformado del siguiente modo: 18 diputados por Guatemala, nueve por El Salvador, seis por Honduras, los mismos por Nicaragua y dos por Costa Rica ${ }^{29}$. La evidencia indica que los representantes de Guatemala ostentaban casi la mayoría en el Congreso, y en consecuencia el poder a la hora de tomar decisiones estaba sensiblemente desequilibrado a su favor.

Sin embargo, este federalismo centralista hubiese ocasionado el inmediato alzamiento de las facciones insurgentes, por lo que se decidió que cada uno de los estados que componían la Federación fuese libre e independiente en su gobierno y administración interior, otorgándoles todo el poder que por la Constitución no estuviese conferido a las autoridades federales. De esta manera el poder legislativo de cada Estado residiría en una Asamblea de representantes elegidos por el pueblo, que quedaría encargada de formar la constitución particular del mismo conforme a la Constitución federal. Mientras que el ejecutivo residiría en un jefe nombrado por el propio pueblo, quien dispondría de su fuerza armada, pudiendo hacer uso de ella para su defensa en caso de invasión, previa comunicación a la Asamblea o en su receso al Consejo.

Como puede apreciarse, el modelo federativo que pretendía implantarse tenía un carácter notablemente diversificado, donde aparte de un poder ejecutivo y legislativo a nivel federal, se delegaba en las diferentes naciones la administración territorial en todas sus facetas, incluida la defensa y disposición de las fuerzas armadas del Estado y la redacción de una Constitución nacional. Por lo tanto, sería el pueblo el que elegiría a sus representantes, y en consecuencia el Presidente y miembros de la Asamblea de cada una de las provincias serían representativos de las demandas políticas del mismo. Por este motivo, si bien las decisiones derivadas del Congreso de la Federación repercutían en todo el istmo vía Constitución de la República, su cuestionamiento e incluso desacuerdo era inevitable en regiones o provincias donde el componente nacionalista era más fuerte. Esta disociación entre los intereses federativos y nacionalistas supuso un impulso más hacia la generalización del contexto bélico en toda la región.

${ }^{29}$ Pérez, 2000, p. 90. Ahora, si nos regimos por los datos del censo de población disponible para 1824, éstos serían: veinte diputados por Guatemala, nueve por El Salvador, cinco por Honduras, seis por Nicaragua y dos por Costa Rica. 
En consecuencia, a pesar de que la Constitución tuvo vigencia hasta marzo de 1840, nunca llegó a consolidar la Federación de Centro América como una pacífica unión de estados. El separatismo de éstos era latente, y el desarrollo federativo se vio indudablemente minado por un conflicto bélico enmarcado en una serie de sucesos que podemos dividir entre los que venían directamente derivados de la estructura colonial, y los que fueron consecuencia del contexto centroamericano de ese momento ${ }^{30}$.

En lo que concierne a los primeros, encontramos en primer lugar la falta de intercambio económico centroamericano. El imperio español nunca estableció comercio o interdependencia económica o comercial alguna entre las diferentes provincias centroamericanas. Todas las colonias fueron organizadas hacia el comercio con la capital del Imperio en una clara estructura de economía colonial, es decir, se exportaban a España materias primas y productos diversos reexportables al resto del mundo, y se enviaba a las colonias productos de mayor valor añadido. No se establecieron intereses comunes intrarregionales, y además las comunicaciones se desarrollaron fundamentalmente hacia los puertos de embarque. A esto hay que añadir que el Imperio se encargaba de impedir cualquier atisbo de diversificación comercial contrario a sus postulados, manteniendo así su monopolio comercial ${ }^{31}$. Por lo tanto, la autosuficiencia no fue un rasgo característico de la economía centroamericana, y su ausencia no propició la constitución de una estructura económica sólida, ni favoreció el surgimiento de grupos sociales ligados a alguna actividad económica importante que se convirtiese en protagonista del desarrollo económico centroamericano ${ }^{32}$.

Debemos también hacer mención a la desproporción existente entre Guatemala y el resto de Centroamérica en lo referente a desarrollo. Recordemos que fue en este país donde se estableció la capital del Reino de Guatemala, y por lo tanto era el verdadero centro de desarrollo regional ${ }^{33}$. Aparte de lo anterior, el gobierno estaba cen-

${ }^{30}$ A estos, FACIO, 1965, pp. 89 y ss. añade uno de carácter estrictamente jurídico: una mejor redacción de la Constitución Federal. Así, piensa que "con la redacción más inteligente de alguno de sus textos, aun hubiera podido retrasarse más la desintegración" de la Federación.

${ }^{31}$ Por ejemplo, se impedía en toda la América española el cultivo del olivo o la vid.

32 Torres-Rivas, 1989, p. 12. Ahora, debido a la evidente revolución que implicó la especialización exportadora de la región con respecto al añil, a lo largo del S. XVIII y hasta la independencia sí podemos hablar de una especialización productiva que, a pesar de ser poco representativa en el marco global, se centraba en el incipiente mercado regional. Así, los cultivos de añil, que se centraban principalmente en la región costera de El Salvador, desplazaron a otras producciones tradicionales como el maíz, el frijol y principalmente la ganadería. En consecuencia, la región se vio en la obligación de importar ganado de sus vecinos para satisfacer la demanda de su población y de su estructura productiva. Por este motivo, Nicaragua, la zona del Pacífico de Honduras y el oriente guatemalteco comenzaron a participar de la economía ganadera. Mientras, la parte occidental de este último, conjuntamente con Chiapas, comenzó una especialización textil que llegó a ser considerada de gran escala --es necesario comentar que la especialización en este sector comenzó realmente en el S. XVI cuando las comunidades indígenas comenzaron a trabajar la lana y el algodón--. Por su parte, la región central de Honduras continuó centrada en la extracción de plata, mientras que Costa Rica, país que presentaba unas exportaciones de cacao en fase terminal, tuvo que recurrir a la explotación de un nuevo producto para mantener su economía. Como consecuencia, el cultivo de tabaco hizo presencia en el istmo, teniendo como máximos demandantes a Nicaragua y el actual Panamá, Solórzano, 1993, p. 34 y ss..

${ }_{33}$ Como ejemplo, se puede hacer mención a que mientras que en el resto de Centroamérica no existían apenas escuelas, en Guatemala había Universidad, colegio de secundaria, consulado de 
tralizado en esta ciudad, lo que ocasionaba que los beneficios obtenidos por la tributación de rentas ingresasen en primer lugar en las arcas del gobierno de España, para posteriormente ser gestionados por el Reino, que priorizaba siempre en su capital ${ }^{14}$. Derivado de este hecho, la población estaba totalmente concentrada, y así, de algo más de un millón doscientos mil habitantes que poblaban en ese momento el istmo, el cincuenta por ciento residía en Guatemala ${ }^{35}$. Esta cuestión, junto a la desarticulación regional y al aislamiento de los estados fruto de las deficientes comunicaciones, afectaba a la representación proporcional y ocasionaba que los estados fuesen reacios a someterse a decisiones de carácter federativo. Así, no es de extrañar que a la hora de elegir entre patria nacional o patria centroamericana, muchos se decidieran por la primera ${ }^{36}$.

Finalmente nos encontramos con unas marcadas diferencias de poder entre las distintas facciones socioeconómicas y con un deficiente sistema tributario que además perjudicaba a los indígenas, lo que provocó sucesivos levantamientos de este colectivo desde 1832 hasta 1837. Paralelamente, a pesar de la mayor participación de los mestizos en las decisiones a nivel regional, el fin de la esclavitud y el auge de libre comercio, la situación de los terratenientes del interior del istmo no cambió sustancialmente y la relación entre señor e indio continuaba marcada por los abusos de poder $^{37}$.

Continuando con los sucesos derivados del contexto centroamericano de ese momento, en lo que respecta a los planes integracionistas de la región, la diplomacia británica, cuya única pretensión era construir un canal interoceánico a través del istmo, siempre se mantuvo cercana a la idea nacionalista de repúblicas independientes. ¿Qué razón la llevó a presentar esta actitud? Indudablemente el hecho de que sus planes imperialistas se centraban en el dominio de la costa caribeña del istmo y en la creación de un canal interoceánico que le asegurase definitivamente el control del comercio marítimo. Por consiguiente era preferible para sus objetivos la creación de cinco pequeñas naciones independientes con las que negociar como imperio que una federación de naciones con una sola voz ${ }^{38}$.

comercio y Administración de Justicia, entre otros. Sol, 1972, p. 67, añade que se podría decir que el desarrollo cultural y económico de este país, para lo que fue el tiempo comprendido entre los siglos XVII y XVIII, era más adelantado que el que tenía Estados Unidos. Tanto así, que la Universidad de San Carlos --aun como Colegio Universitario de Santo Tomás-- fue fundada con anterioridad a la de Harvard (1562 y 1636, respectivamente).

${ }^{34}$ Los ingresos tributarios provenían fundamentalmente de las aduanas y del consumo de tabaco y alcohol..

${ }^{35}$ Según cifras extraídas de Sol, 1972, y Gudmundsun, 1993.

${ }^{36}$ A este respecto, la pregunta que subyace en todo el trabajo de TARACENA, 1995, es si realmente podemos hablar de algún tipo de sentimiento nacional centroamericano; mientras que el caso de Collado et al., 1986, la cuestión tiene relación con el interrogante de si después de tres siglos de colonialismo verdaderamente la región tenía unas raíces lo suficientemente firmes como para que se mantuviesen después de la independencia. TORRES-RIVAS, 1989, incluso se atreve a afirmar que la región se encontraba centralizada como Reino por razones de exclusiva conveniencia administrativa.

${ }^{37}$ Gudmundsun, 1995.

${ }^{38} \mathrm{La}$ era de las presiones diplomáticas se inauguró en 1825 cuando la República Federal, vía Congreso, firmó un primer empréstito con la Casa Barclays, Herring \& Richardson de Londres por 
Sin embargo, el que consideramos principal hecho de estas características se concreta en las diferencias ideológicas entre conservadores y liberales, que sumieron a la región en un conflicto bélico que se alargó durante varios años. En un ejercicio de demarcación política, los primeros siempre habían sido partidarios de la unidad con España, por lo que la independencia supuso un duro golpe para una facción política que defendía una fuerte influencia de la iglesia dentro de un Estado totalmente centralizado. Por su parte, los liberales, encabezados por Manuel José Arce y el general hondureño Francisco Morazán, plantaron cara a un sector conservador que, encabezados por la iglesia guatemalteca y los grandes comerciantes de la capital, se oponía a la reforma constitucional que buscaba el restablecimiento de la autoridad efectiva en el Gobierno Federal ${ }^{39}$. Toda esta lucha entre facciones ideológicas culminó en 1829 cuando las cuatro provincias se unieron contra Guatemala y llevaron a Morazán a la Presidencia de la República de Centroamérica. Pero a partir de esta fecha el contexto bélico impidió cualquier tipo de decisión a nivel federal, y el sueño unionista concluyó cuando en 1838 los gobiernos de Nicaragua, Costa Rica y Honduras deciden separarse de una Federación que nunca supo como unir a la región.

\section{CONCLUSIONES}

Tras concluir este episodio histórico podemos ya responder a las cuestiones planteadas al inicio de este trabajo. Así, en relación a las causas que ocasionaron la independencia de las provincias del antiguo Reino de Guatemala, la evidencia indica la casi imposibilidad del mantenimiento del régimen colonial. En todo el continente americano los levantamientos independentistas habían dejado claro la poca consistencia del mismo, a la par que la oligarquía criolla demandaba un puesto preferente en la historia del istmo. En ese sentido, los alzamientos militares comandados por Bolívar y San Martín pusieron en entredicho de manera definitiva la dominación de la metrópoli, y sentaron las bases de la emancipación de todos los países integrantes del imperio español. Sin embargo, es importante señalar que en el caso del istmo centroamericano las provincias afectadas no tuvieron que recurrir al conflicto armado o a la rebelión social.

un monto de 5 millones de pesos. A partir de este momento, esa dependencia financiera se convertiría en una verdadera debilidad regional que daría pretextos al cónsul británico Frederick Chatfield para amenazantes reclamos del pago de intereses combinados con manifiestas ambiciones sobre la costa del Caribe, Pérez, 2000, p. 93. En Torres-Rivas 1989, pp. 17 y ss., se expone la evolución de esta deuda, remarcando que la cancelación de la misma se extendió décadas, llegando incluso hasta mediados del siguiente siglo en el caso de Honduras y Nicaragua.

${ }^{39}$ Los grupos sociales más conservadores se localizaban ante todo en las antiguas capitales de provincia: León, Comayagua, Cartago y fundamentalmente Ciudad de Guatemala. Mientras que la élite social que abrazaba ideales independentistas y republicanos se localizaba principalmente en la región salvadoreña, pero también, aunque con menor peso, en Granada, San José, Tegucigalpa y en la propia provincia de Guatemala. Este último grupo estaba conformado por agrupaciones de terratenientes de todas las regiones comentadas, así como comerciantes en el caso de Granada, mineros en el caso de Tegucigalpa, medianos propietarios en el caso de San José y Guatemala, y funcionarios e intelectuales que se centraban fundamentalmente en esta última ciudad. PinTo, 1993, p. 74. 
En este caso la emancipación fue pacífica y lógica, al venir de la mano de la propuesta planteada por el imperio mexicano. Es decir, no hubo transición violenta ni ejércitos enfrentados. Solamente burocracia y un claro juego político por parte de la clase social dominante -la oligarquía- que planeó la evolución de la región con la lógica de lo que estaba ocurriendo en todos los países vecinos.

Pero, ¿quiere esto decir que el contexto social reinante era pacífico y democrático? A pesar de que no se dio en la región un conflicto entre ejércitos regulares claramente demarcados, el alzamiento social y los enfrentamientos armados proliferaron en toda el área. Desde los episodios de El Salvador y Guatemala de 1811, el conflicto entre las masas y las milicias institucionales fue continuo ${ }^{40}$. Este hecho obliga a completar el análisis con un estudio de clases sociales que puede ayudar a enfocar este episodio desde otro punto de vista, pues a pesar de que la elite criolla en todo momento mantuvo las riendas del proceso, no fue la única protagonista y los hechos comentados obligan a ampliar el marco de análisis hacia otros protagonistas que algunos investigadores denominan "subalternos"

Esta última aclaración nos lleva a la segunda de las cuestiones planteadas: la relacionada con las élites sociales. Así, el mantenimiento del poder y del orden colonial se convirtió en una prioridad para un sector que veía en el proceso de independencia y en las propuestas liberales una amenaza a sus privilegios. La oligarquía colonial que ostentaba el poder en Centroamérica -principalmente criollos y españoles residentes en Guatemala- en ningún momento dio opción a la sociedad civil para tomar decisiones de tipo federal. Siempre se mantuvo firme ante revueltas sociales e incluso ante alzamientos de tinte oligárquico llevados a cabo en provincias insurgentes. Por consiguiente, la proclamación de la independencia y la posterior adhesión al Plan de Iguala no fueron más que las dos caras de una misma moneda, y la suscripción de la Constitución Federal y el fallido intento de la Federación una nueva herramienta de control político.

¿Qué importante deducción podemos extraer de lo que acabamos de comentar? El fuerte carácter endógeno del proceso de independencia centroamericano. Generalmente se suele argumentar que la independencia fue forzada desde el exterior, importada, o dicho de otro modo, que tuvo un marcado carácter exógeno al venir de la mano de Agustín de Iturbide y la adhesión al Plan de Iguala. Sin embargo, como hemos podido apreciar a lo largo de este trabajo, es evidente que no podemos dejar de lado los condicionantes endógenos que han marcado la evolución del proceso. Así, a la decadencia de la metrópoli y a las deficiencias estructurales derivadas de la ausencia de un plan de desarrollo económico regional por parte de la corona española, se unió la centralización del gobierno y de la gestión del Reino en una sola provincia. Ambas sirvieron de estímulo al surgimiento de las ideas libertarias y nacionalistas que esgrimieron tanto las elites locales como los grupos sociales liberales. La búsqueda de la independencia en este caso fue ajena a la invitación del imperio vecino, pues se forjó con anterioridad y como inferencia de una estructura colonial completamente inoperante. Con o sin invitación exterior, la presión sociopolítica interna hacía insostenible el modelo de gestión.

40 Además, a lo anterior hay que unir los numerosos levantamientos del pueblo indígena, fundamentalmente en Guatemala.

${ }^{41}$ Pollack, 2008 y 2009. 
Pero también es cierto que con el nacimiento de la Federación no se solventaron todos los problemas estructurales que atesoraba el istmo, y que finalmente la llevaron a su destrucción. La pregunta que emana de la anterior afirmación es si, con una consistente política de cohesión y desarrollo regional, hubiese sido posible que la Federación de Centro América se convirtiera en una realidad. A este respecto pensamos que los importantes condicionantes liberales y nacionalistas, junto con la escasa preparación política para llevar a cabo políticas de este tipo, hicieron imposible la puesta en práctica de mecanismos eficientes de integración. Es decir, en cierta manera, con el nacimiento de la Federación se firmaba su sentencia, pues la región quizá no se encontrase aun preparada para llevar a cabo una exitosa convivencia que exigía de ciertas políticas de integración regional imposibles de llevar a cabo en ese momento. En la Constitución no se contemplaba ningún tipo de herramienta de este tipo, ni se mencionaba como propósito la necesidad tanto de potenciar el desarrollo de la región en conjunto como de hacerlo en las regiones donde la evidencia así lo exigía. Así mismo, no se contemplaba plan económico alguno y simplemente se delegaba la gestión de cada uno de los estados en su propia Administración, sin que se explicitasen directrices de política a seguir ni propósitos generales de carácter económico, político y social.

Queda claro que en tales circunstancias la creación de una Federación sin un plan de política económica y social consistente hacía imposible el ideal reflejado en la Constitución de la República Federal. Sin lo anterior, entramos en el ámbito de los deseos, pero nos alejamos de la realidad. La no correspondencia entre las condiciones materiales y el ideal liberal que implicaba una nación igualitaria labrada mediante el consenso entre las diferentes clases y grupos sociales, fue un impedimento insalvable que se vio además potenciado por la impaciencia nacionalista de algunos de los países implicados, que a la hora de elegir entre el contexto nacional y el regional se decidieron sin dudar por el primero.

\section{REFERENCIAS BIBLIOGRÁFICAS}

ACUÑA, Víctor Hugo

2002 "La invención de la diferencia costarricense, 1810-1870". Revista de Historia. San José. no 45, pp. 191-228.

2005 "Concepciones de la comunidad política en Centroamérica en tiempos de la independencia, (1820-1823)". En Colom, Francisco (ed.). Relatos de nación. La construcción de las identidades nacionales en el mundo hispánico. 2 Vols. Madrid - Frankfurt. Iberoamericana - Vervuert, pp. 251-274.

Alda, Sonia

2000 La participación indígena en la construcción de la república de Guatemala, S. XIX. Madrid. Universidad Autónoma de Madrid. 
AnNA, Timothy

1991 "La Independencia de México y América Central” [1985]. En BethelL, Leslie (coord.). Historia de América Latina. Vol. 5. Barcelona. Crítica, pp. 41-74.

2003 "Casos de continuidad y ruptura: Nueva España y Capitanía General de Guatemala”. En CARrera DAMAs, Germán - Lombardi, John (coords.). Historia General de América Latina. Vol. 5. La crisis estructural de las sociedades implantadas. París. Trotta - UNESCO, pp. 207-238.

AvendaÑo, Xiomara

1995 "Elecciones, ciudadanía y representación política en el Reino de Guatemala, 1810-1821". Boletín AFEHC. n 12. Documento digital disponible en: http://afehc-historia-centroamericana.org/index. php?action=fi_aff\&id=364.

2007 "La independencia de Guatemala y El Salvador: una nueva visión sobre los actores". En Chust, Manuel - Serrano, Antonio (eds.). Debates sobre las independencias iberoamericanas. Madrid - Frankfurt. Iberoamericana - Vervuert, pp. 237-254.

2008 "Las instrucciones para elegir diputados al primer congreso constituyente mexicano: un intento para controlar a las provincias". Boletín AFEHC. $\mathrm{n}^{\mathrm{o}}$ 34. Documento digital disponible en: http://afehc-historiacentroamericana.org/.

Benson, Nettie L. y Berry, Charles

1969 "The Central American delegation to the First Constituent Congress of Mexico, 1822-1824". Hispanic American Historical Review. Durham. vol. 49: 4, pp. 679-701.

BERTRAND, Michel

1987 Terre et société coloniale: les communautés maya-quichés de la région du Rabinal du XVIème au XIXème siècles. Mexico. CEMCA.

CAÑas-Dinarte, Carlos

2002 Centroamérica: una historia de uniones y desuniones. Documento digital disponible en: http://www.sgsica.org.

CArdoso, Ciro y Pérez brignoli, Héctor

1977 Centroamérica y la economía occidental (1520-1930). Costa Rica. Editorial de la Universidad de Costa Rica.

CARvalho, Alma Margarita

1994 La ilustración del despotismo en Chiapas. México. Dirección General de Publicaciones del CONACULTA.

CERDAs, Rodolfo

1967 La formación del Estado en Costa Rica (1821-1842). Costa Rica. Publicaciones de la Universidad de Costa Rica. 
Chust, Manuel y Serrano, José Antonio

2007 "Nueva España versus México: historiografía y propuestas de discusión sobre la Guerra de Independencia y el Liberalismo doceañista". Revista Complutense de Historia de América. Madrid. n 33, pp. 15-33.

Collado, Carmen; Dutrenit, Silvia ; Gullén, Diana; López, Selva y Yankelevich, Pablo (coords.) 1988 Centroamérica, Textos de la Historia de Centroamérica y el Caribe. Vols. 1 y 2. México. Instituto de Investigaciones Dr. José María Luis Mora-Universidad de Guadalajara- Secretaría de Educación Pública.

Collado, Carmen; Dutrenit, Silvia; Guillén, Diana; Toussaint, Mónica y Yankelevich, Pablo 1986 "Continuidad y cambio en la historia centroamericana". Secuencia, $\mathrm{n}^{\circ} 4$, enero-abril, pp. 102-113.

Dalton, Roque

1984 El Salvador [1965]. México. Universidad Autónoma de Puebla.

DемYк, Noelle

1995 "Los territorios del Estado-Nación en América Central. Una problemática regional". En TARAcena Arriola, Arturo - Piel, Jean (comps.). Identidades nacionales y Estado Moderno en Centroamérica. San José. Editorial de la Universidad de Costa Rica, pp. 13-30.

Díaz, David

2002 "La invención de las Naciones en Centroamérica, 1821-1950". Coloquio

Identidades Revis(it)adas, artes visuales, literatura, música, danza e historia en América Central. Managua - Nicaragua. Instituto de Historia de Nicaragua y Centroamérica de la Universidad Centroamericana, Centro de Investigación en Identidad y Cultura Latinoamericanas de la Universidad de Costa Rica y Servicio Alemán de Intercambio Académico de la Iniciativa Cultural Alemana. Documento digital disponible en la página web de la AFEHC: http:// ress.afehc.apinc.org/articulos2/fichiers/portada_afehc_articulos14.pdf

FACIO, Rodrigo

1938 "Esquema social de la Independencia". Revista de los Archivos Nacionales. San José. vol. 2, pp. 178-195.

1965 La Federación de Centroamérica. Sus antecedentes, su vida y su disolución. San José. ESAPAC.

FALLA, Ricardo

1971 "Actitud de los indígenas de Guatemala en la época de la Independencia 1800-1850. El problema de los límites entre las comunidades de Santa María Chiquimula y San Antonio Ilotenango". Estudios Centroamericanos. Revista de la Universidad Centroamericana José Simeón Cañas. San Salvador. nº. 278, pp. 702-718. 
FERNÁNDEZ, José Antonio

2003 "De tenues lazos a pesadas cadenas. Los cabildos coloniales en El Salvador como arena de conflicto". En Herrera, Sajid Alfredo - GómEz, Margarita (comp.). Mestizaje, poder y sociedad. Ensayos de historia colonial de las provincias de San Salvador y Sonsonate. El Salvador. FLACSO, pp. 73-95.

FERNÁNDEZ, Ricardo

1971 Historia de Costa Rica. La Independencia. Costa Rica. Universidad de Costa Rica.

FLOYD, Troy S.

1989 "Los comerciantes guatemaltecos, el gobierno y los provincianos, 17501800". En CÁCERES, Luis René (ed.). Lecturas de Historia de Centroamérica. Costa Rica. BCIE-EDUCA, pp. 177-197.

FonsECA, Elizabeth

1998 Centroamérica: su historia. Costa Rica. FLACSO-Costa Rica, EDUCA.

FRASQUET, Ivana

2007 "La "otra" independencia de México: el primer imperio mexicano. Claves para la reflexión histórica". Revista Complutense de Historia de América. Madrid. no 33, pp. 35-54.

GARCíA, Jorge Mario

1994 Centroamérica en las Cortes de Cádiz. México. Fondo de Cultura Económica. [Primera edición, Costa Rica, EDUCA, 1971, como: Orígenes de la democracia constitucional en Centroamérica].

GuARISCO, Claudia

2007 "La Constitución de Cádiz y la participación política popular en la Nueva España, 1808-1821. Balance y nuevas perspectivas". Revista Complutense de Historia de América. Madrid. nº 33, pp. 55-70.

GuDMUNDSUN, Lowell

1993 "Sociedad y política (1840-1871)". En Pérez Brignoli, Héctor (coord.) De la ilustración al liberalismo (1750-1870). Tomo III de la Historia general de Centroamérica. Madrid. Sociedad Estatal Quinto Centenario y Facultad Latinoamericana de Ciencias Sociales. pp. 203-256.

1995 "Señores y campesinos en la formación de la Centroamérica moderna. La tesis de Barrington Morre y la historia centroamericana". En Taracena Arriola - Piel (comps.), pp. 31-41.

Guerra, Francois-Xavier

1992 Modernidad e independencias. Ensayo sobre las revoluciones hispánicas. Madrid. Encuentro. 
Gutiérrez Álvarez, Coralia

2009 "La historiografía contemporánea sobre la independencia en Centroamérica". Nuevo Mundo Mundos Nuevos. Debates. Documento digital puesto en línea el 03 febrero 2009, disponible en: http:// nuevomundo.revues.org/54642.

GutiÉRreZ, Sergio N.

2005 Chiapas histórico. De la independencia a la revolución 1821-1920. México. Gobierno del Estado de Chiapas. Secretaría de Educación.

Hall, Carolyn y Pérez, Héctor

2003 Historical Atlas of Central America. Norman. University of Oklahoma Press.

HerRarte, A (comp.)

1956 Documentos de la unión Centroamericana. Guatemala -ODECAEditorial del Ministerio de Educación Pública.

KINLOCH, Frances

1999 Nicaragua: identidad y cultura política. Managua.. Banco Central de Nicaragua.

KRIEDTE, Peter

1994 Federalismo tardío y capital mercantil [1982]. Barcelona. Crítica.

Lindo-FuENTES, Héctor

1993a "Economía y sociedad (1810-1870)". En Pérez Brignoli (coord.), pp. 141-201.

1993b "Consecuencias económicas de la independencia en Centroamérica". En Prados de la Escosura, Leandro - Amaral, Samuel (eds.). La independencia americana: consecuencias económicas. Madrid. Alianza Editorial, pp. 54-79.

LuJÁN, Jorge

1982 La independencia y la anexión de Centroamérica a México [1975]. Guatemala. Editorial Serviprensa Centroamericana.

LYNCH, John

1973 Las revoluciones hispanoamericanas, 1808-1826. Barcelona. Crítica.

2001 América Latina, entre ciudadanía y nación. Barcelona. Crítica.

MARroquín, Alejandro D.

1964 Apreciación sociológica de la independencia salvadoreña. San Salvador. Instituto de Investigaciones Económicas de la Facultad de Ciencias Económicas de la Universidad de El Salvador. 
Martínez Peláez, Severo

1979 La patria del criollo [1970]. Costa Rica. EDUCA.

S.f. Centroamérica en los años de la independencia: El país y los habitantes. Guatemala. IIES, USAC.

MaruRe, Alejandro

1960 Bosquejo histórico de las Revoluciones de Centroamérica. Desde 1811 hasta 1834 [1837]. Guatemala. Editorial del Ministerio de Educación Pública "José de Pineda Ibarra".

MAYES, Guillermo

1956 Honduras en la Independencia. Tegucigalpa. Tipografía Nacional.

MELÉnDEZ, Carlos

1970 La ilustración en el Reino de Guatemala. Costa Rica, EDUCA.

1993 La Independencia de Centroamérica. Madrid. Mapfre.

Mitre, Bartolomé

1950 Historia de San Martín y de la emancipación sudamericana [1887]. Buenos Aires. El Ateneo.

Molina, Iván

2002 Costarricense por dicha. Identidad nacional y cambio cultural en Costa Rica durante los siglos XIX y XX. San José. Editorial de la Universidad de Costa Rica.

OBREGÓn, Rafael

1971 De nuestra historia patria. San José. Universidad de Costa Rica.

1979 Costa Rica en la Independencia y La Federación. San José. Editorial Costa Rica.

Palma, Gustavo

1986 "Núcleos de poder local y relaciones familiares en la ciudad de Guatemala a finales del siglo XVIII". Mesoamérica. Guatemala. $\mathrm{n}^{\circ} 12$, diciembre, pp. 241-308.

Pérez Brignoli, Héctor

2000 Breve historia de Centroamérica. Madrid. Alianza Editorial.

Pérez BRIgnoli, Héctor (coord.)

1993 De la ilustración al liberalismo (1750-1870). Tomo III de la Historia general de Centroamérica. Madrid. Sociedad Estatal Quinto Centenario y Facultad Latinoamericana de Ciencias Sociales.

Pinto, Julio César

1986 Centroamérica, de La Colonia al Estado Nacional 1800-1840. Guatemala. Editorial Universitaria.

1993 "La independencia y la federación (1810-1840)". En Pérez Brignoli (coord.), pp. 73-140. 
Pollack, Aaron

2008 Levantamiento K'iche' en Totonicapán. Los lugares de la política subalterna. Guatemala. AVANCSO.

2009 "Las Cortes de Cádiz en Totonicapán: una alianza insólita en un año insólito". Stvdia Histórica. Salamanca. vol. 27, pp. 207-234.

QuesADA, Juan Rafael

2002 Historia de la historiografia costarricense. San José. Editorial de la Universidad de Costa Rica.

RODRÍGUEZ, Mario

1984 El experimento de Cádiz en Centroamérica. 1808-1826 [1978]. México. Fondo de Cultura Económica.

SAMAYOA, Héctor Humberto

1960 Implantación del régimen de intendencias. Guatemala. José De Pineda Ibarra.

1972 Ensayos sobre la independencia de Centroamérica. Guatemala. Editorial Universitaria.

SIERRA, Rolando

2003 Honduras como interpretación. Tegucigalpa. PNUD.

Sol, Jorge

1972 "Proceso de la integración económica centroamericana". Revista de la Integración Centroamericana. Tegucigalpa. $\mathrm{n}^{\circ}$ 4, pp. 45-100.

SolóRzAno, Juan Carlos

1993 "Los años finales de la dominación española (1750-1821)". En Pérez BRIGNOLI (coord.), pp. 13-72.

Soto-Quirós, Ronald

2006 "Reflexiones sobre el mestizaje y la identidad nacional en Centroamérica: de la colonia a las Repúblicas liberales". Boletín AFEHC. n 25, pp. 1-40.

Taracena Arriola, Arturo

1995 "Nación y República en Centroamérica (1821-1865)". En TARACENA Arriola - Piel (comps.), pp. 45-61.

1997 Invención criolla, sueño ladino, pesadilla indígena. Los Altos de Guatemala: de región a Estado, 1740-1850. San José de Costa RicaGuatemala. Editorial Porvenir, CIRMA, Cooperación Técnica y Científica del Gobierno de Francia.

2002 Etnicidad, estado y nación en Guatemala, 1808-1944. Vol. I. Guatemala. CIRMA.

Taracena Arriola, Arturo y Piel, Jean (comps.)

1995 Identidades nacionales y Estado Moderno en Centroamérica. San José. Editorial de la Universidad de Costa Rica. 
TORRES-Rivas, Edelberto

1989 Interpretación del desarrollo social centroamericano [1971]. San José. Facultad Latinoamericana de Ciencias Sociales.

2007 La piel de Centroamérica. San Salvador. Madrid. Facultad Latinoamericana de Ciencias Sociales.

Toussaint, Mónica; Rodríguez, Guadalupe y VÁzQuez, Mario

2001 Vecindad y diplomacia. Centroamérica en la política exterior mexicana 1821-1988. México. SER.

Townsed, Andrés

1973 Las provincias unidas de Centro América. Fundación de la República. San José de Costa Rica. EDUCA.

Vives, Pedro

1987 "Intendencias y poder en Centroamérica, la reforma incautada". Anuario de Estudios Centroamericanos. San José de Costa Rica. vol. 13: 2, pp. 37-47.

WOODWARD, Ralph Lee Jr.

1976 Central America. A Nation Divided. New York. Oxford University Press.

1989 "Los comerciantes guatemaltecos". En CÁcEREs, Luis René (ed.). Lecturas de Historia de Centroamérica. Costa Rica. BCIE-EDUCA.

WORTMAN, Miles

1976 "Legitimidad política y regionalismo: el Imperio mexicano y centroamericano". Historia Mexicana. México. vol. 26: 2, pp. 238-262.

1991 Gobierno y sociedad en Centroamérica 1680-1840. San José de Costa Rica. BancoCentroamericano de Integración Económica, EDUCA.

Zelaya, Chester

1971 Nicaragua en la Independencia. San José deCosta Rica. Editorial Universitaria Centroamericana.

Zilberman, Cristina (ed.)

1994 Siglo XVIII hasta la Independencia. En LuJÁn, Jorge (ed.). Historia general de Guatemala. Tomo 3. Guatemala. Asociación de Amigos del País - Fundación para la Cultura y el Desarrollo. 